\section{Istituto}

Nazionale

Fisica

Nucleare
Sezione SANITÀ

Istituto Superiore di Sanità

Viale Regina Elena 299

I-00161 Roma, Italy

INFN-ISS 97/12

October 1997

\title{
Can neutron electromagnetic form factors be obtained by polarized inclusive electron scattering off polarized three-nucleon bound states?
}

\author{
A. Kievsky ${ }^{a}$, E. Pace ${ }^{b}$, G. Salmè ${ }^{c}$ and M. Viviani ${ }^{a}$ \\ ${ }^{a}$ INFN, Sezione di Pisa, 56010 S.Piero a Grado, Pisa, Italy \\ ${ }^{b}$ Dipartimento di Fisica, Università di Roma "Tor Vergata" and Istituto Nazionale di Fisica \\ Nucleare, Sezione Tor Vergata, Via della Ricerca Scientifica 1, I-00133 Roma, Italy \\ ${ }^{c}$ INFN, Sezione Sanità, Viale Regina Elena 299, I-00161 Roma, Italy
}

\begin{abstract}
The investigation of the electromagnetic inclusive responses of polarized ${ }^{3} \mathrm{He}$ within the plane wave impulse approximation is briefly reported. A particular emphasys is put on the extraction, from the inclusive responses at the quasielastic peak, of the neutron form factors from feasible experiments.
\end{abstract}

Presented to XVth International Conference on "Few-body problems in Physics", Groningen, July 1997. To appear in Nucl. Phys. A 


\section{INTRODUCTION}

In the past few years the possibility of extracting the neutron electromagnetic (em) form factors from measurements of inclusive scattering of polarized electrons by polarized ${ }^{3} \mathrm{He}$ has attracted great attention [1]. Unfortunately, a reliable determination of neutron form factors is prevented by many difficulties, primarily by the presence of the proton and the effect of the final state interaction (FSI) and possibly by meson exchange currents, isobar components in the target wave function and relativistic effects. In particular the overwhelming role played by the proton in the transverselongitudinal response has not allowed an accurate estimate of the neutron electric form factor at low momentum transfer.

Our investigation [2] has been focused on the possible extraction of both the electric and magnetic neutron form factors from the inclusive polarized cross sections within the Plane Wave Impulse Approximation (PWIA). We restricted the analysis at the quasielastic peak, where FSI should play a minor role (cf. the preliminary calculations including FSI shown in [3]) and we considered a range of momentum transfer up to $2(\mathrm{GeV} / \mathrm{c})^{2}$, namely the kinematical region relevant for TJNAF [ [⿴囗十]].

\section{RESULTS}

The main ingredient of our calculations is a refined spin-dependent spectral function [2] of the three-nucleon system. Various spectral functions corresponding to different, realistic two- and three-body nuclear forces [5, 8] have been considered. As well known [9, [10] the spin-dependent spectral function yields the probability distribution of finding a nucleon, inside a nucleus, with given polarization, three-momentum and removal energy. First of all, we have analyzed in detail, at the qe peak, the model dependence upon the initial state interaction of the inclusive responses as a function of $Q^{2}$, obtaining a very encouraging result (from the point of view of a model independent determination of the neutron form factors): the changes due to a different choice of two- and three-body forces and to the Coulomb interaction as well, are of the order of a few percent. In Fig. 1, the calculations illustrating this result for the transverse, $R_{T^{\prime}}^{3 H e}$, and the transverse-longitudinal, $R_{T L^{\prime}}^{3^{H}}$ responses at the qe peak are shown (the Galster [1]] nucleon form factors have been adopted). It is worth noting that the Brazil [7] and the Tucson-Melbourne [8] three-body interactions essentially yield the same results.

After investigating the effects of changing the interaction in the initial state we have analyzed the proton contribution. It should be pointed out that the presence of the proton has a quite different effect in the inclusive responses of polarized ${ }^{3} \mathrm{He}$ : in particular in the transverse response it is $\approx 10 \%$, while in the transverse-longitudinal one it ranges between $80 \%$, at low values of $Q^{2}$, and $40 \%$, at the highest values. These features are illustrated in Fig. 2a. In Fig. 2b, for the sake of comparison, the same analysis is shown for the polarized ${ }^{3} \mathrm{H}$, that represent a very good effective proton target, while the corresponding statement "the polarized ${ }^{3} \mathrm{He}$ is an effective neutron target" should be considered with some care for the case of $R_{T L^{\prime}}^{3}$. Nevertheless the proton predominance can yield an unexpected help if the PWIA represents a reliable approximation for $R_{T L^{\prime}}^{3}$ at the qe peak (cf. [3]). As a matter of fact, as it was shown 

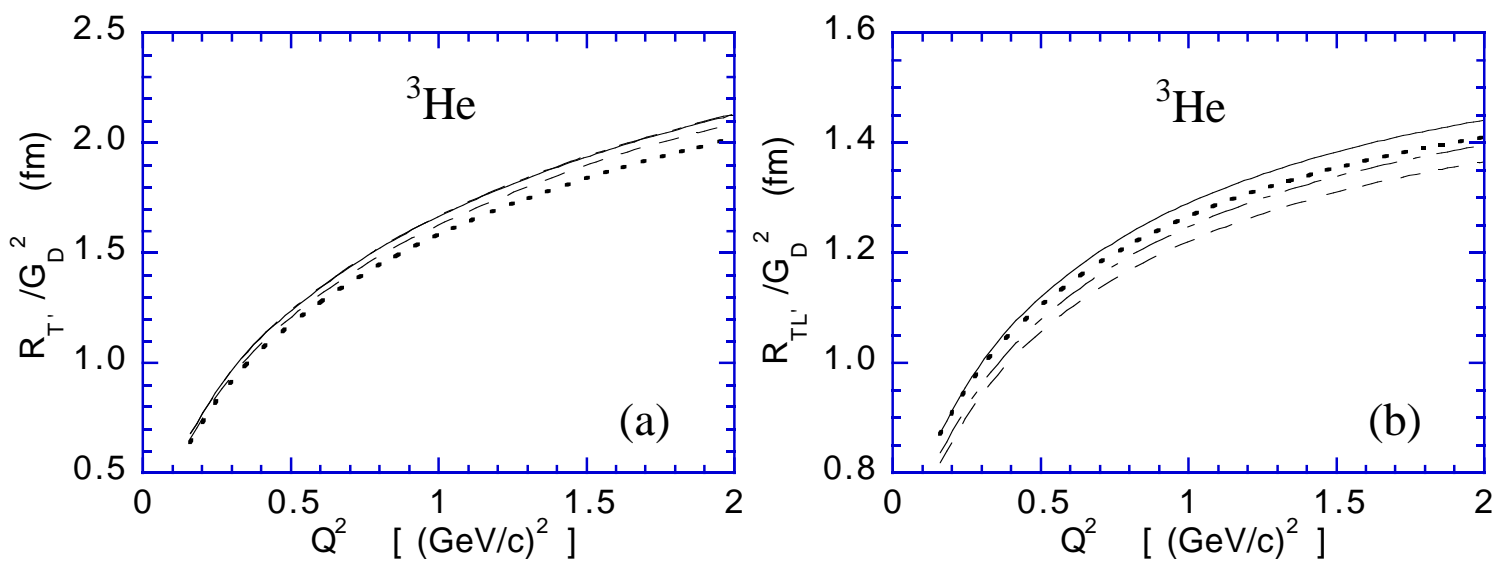

Figure 1. a) The ratio $R_{T^{\prime}}^{3}\left(Q^{2}, \nu_{\text {peak }}\right) / G_{D}^{2}\left(Q^{2}\right)$ vs $Q^{2}\left(G_{D}\left(Q^{2}\right)=1 /\left(1+Q^{2} / 0.71\right)^{2}\right)$. Solid line: $R_{T}$ obtained from AV14 [5] + Coulomb interaction; dot-dashed line: the same as the solid line, but without Coulomb interaction, (this line largely overlaps with the solid one); dotted line: the same as the solid line, but for RSCv8 potential [6]; dashed line: the three-body interaction [7, 8] has been added to Av14 + Coulomb interaction (see text). b) The same as Fig. 1(a), but for $R_{T L^{\prime}}^{3 H e}\left(Q^{2}, \nu_{\text {peak }}\right)$. (After Ref. 2)

in [2] $\partial\left[R_{T L^{\prime}}^{3} / G_{M}^{p} G_{E}^{p}\right] / \partial Q^{2}$ is almost constant at low momentum transfer $\left(0.1 \leq Q^{2} \leq 0.3\right.$ $\left.(\mathrm{GeV} / \mathrm{c})^{2}\right)$, and it is proportional to a neutron structure function. This latter quantity contains only the neutron spin-dependent spectral function, without any em form factor dependence (the same feature occurs for the corresponding proton structure function). In Fig. 3, the ratio of the polarized transverse-longitudinal response and the proton form factors, i.e. $R_{T L^{\prime}}^{3 H e}\left(Q^{2}, \nu_{\text {peak }}\right) /\left(-2 \sqrt{2} G_{M}^{p}\left(Q^{2}\right) G_{E}^{p}\left(Q^{2}\right)\right)$, is presented. It should be pointed that a constant behaviour of the proton contribution to such a ratio is predicted by the PWIA, while the linear behaviour in $Q^{2}$ for the neutron one is the combined effect of the almost constant value of the neutron structure function (as shown by PWIA calculations) and the general property of the electric neutron form factor to be linearly vanishing for $Q^{2} \rightarrow 0$. The comparison with the experimental data can allow one to extract the proton contribution to $R_{T L^{\prime}}^{3}$ and then to isolate the neutron one. Furthermore the comparison could give valuable information on the extent to which i) PWIA holds and ii) the extraction of the neutron form factors can be achieved (or in other words, the factorization of the nucleon structure functions can be applied [2]).

One can also determine experimentally the proton contribution to $R_{T^{\prime}}^{3}$. In fact, a measurement of the ratio $R_{T^{\prime}}^{p}\left(Q^{2}, \nu_{\text {peak }}\right) / R_{T L^{\prime}}^{p}\left(Q^{2}, \nu_{\text {peak }}\right)$ can be obtained through an accurate determination of the polarization angle $\beta_{\text {critic }}$, where the proton contribution to the polarized cross section, is vanishing. An estimate of such an angle, at the qe peak, can be obtained by measuring the integrated amount of protons knocked out along the direction of $\vec{q}$, since at the qe peak protons are emitted preferably along such a direction. In principle this is an exclusive measurement, but it can be 

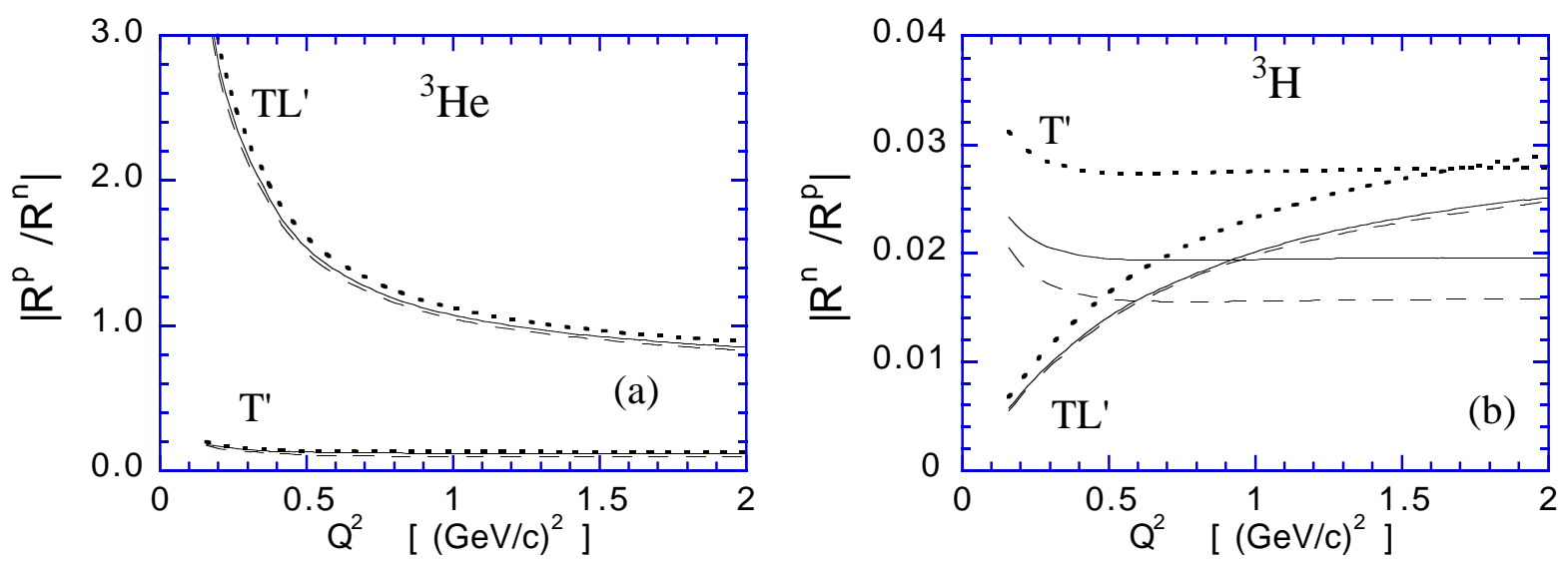

Figure 2. a) The ratio $R_{p} / R_{n}$ in ${ }^{3} \mathrm{He}$ vs $Q^{2}$, for the transverse-longitudinal $\left(T L^{\prime}\right)$ and the transverse $\left(T^{\prime}\right)$ responses. Solid lines: inclusive responses obtained from AV14 + Coulomb interaction; dotted lines: the same as the solid lines, but for RSCv8 potential; dashed lines: the three-body interaction has been added to Av14 + Coulomb interaction. b) The same as Fig. 2(a), but for $R_{n} / R_{p}$ in ${ }^{3} \mathrm{H}$

performed more easily than the one where knocked out neutrons are measured since i) only protons have to be detected and ii) row data are sufficient for estimating $\beta_{\text {critic }}$ (one has only to determine the polarization angle where the polarized contribution to the inclusive cross section changes sign). Finally, the PWIA prediction of this angle could be used as a reliable guideline for the experimental measurements, since $\beta_{\text {critic }}$ depends upon the ratio of responses, and therefore it should be less sensitive to various effects, such as FSI, than each response separately.

\section{SUMMARY}

We have proposed distinct measurements that allow the extraction of the neutron contribution to the total responses and then an estimate of the ratio $G_{E}^{n} / G_{M}^{n}$. Moreover, by introducing theoretical calculations of the nuclear structure functions one could even obtain $G_{E}^{n}$ and $G_{M}^{n}$ separately. It should be emphasized that a measurement of the em inclusive responses of polarized ${ }^{3} \mathrm{H}$ could allow one to check more directly the reaction mechanism, in particular the factorization of the responses at the qe peak (essential for extracting the neutron form factors), and to obtain the nuclear structure functions. Once such quantities were available, the model dependence in the extraction of $G_{E}^{n}$ and $G_{M}^{n}$ could be substantially lowered by using the ${ }^{3} \mathrm{H}$ structure functions as an experimental estimate of the corresponding quantities for ${ }^{3} \mathrm{He}$.

\section{References}




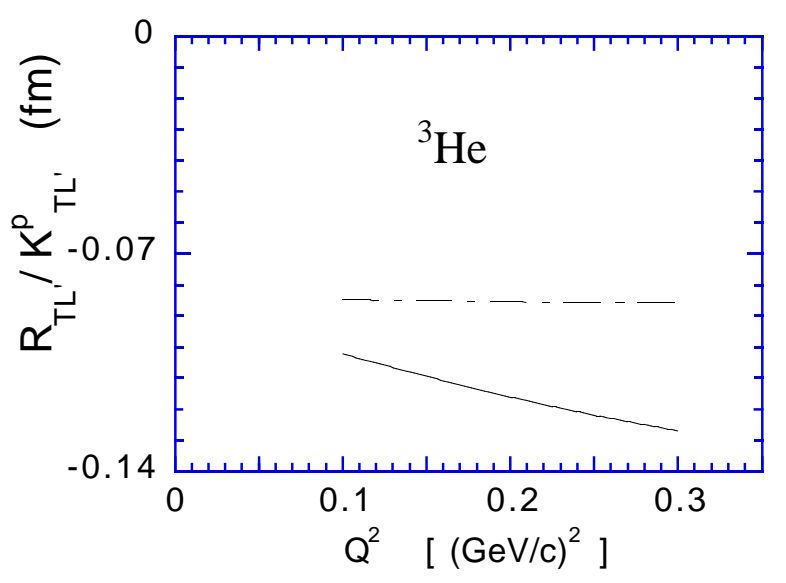

Figure 3. The ratio $R_{T L^{\prime}}^{3}\left(Q^{2}, \nu_{\text {peak }}\right) / K_{T L^{\prime}}^{p}$, with $K_{T L^{\prime}}^{p}=-2 \sqrt{2} G_{E}^{p} G_{M}^{p}$, vs $Q^{2}$. Solid line: result obtained by the spin-dependent spectral function corresponding to Av14 + Coulomb interaction and the Galster nucleon form factors [11]. Dot-dashed line: $R_{T L^{\prime}}^{3}{ }^{3}{ }^{\prime}, p K_{T L^{\prime}}^{p}$, shown as a reference line. (After [2]).

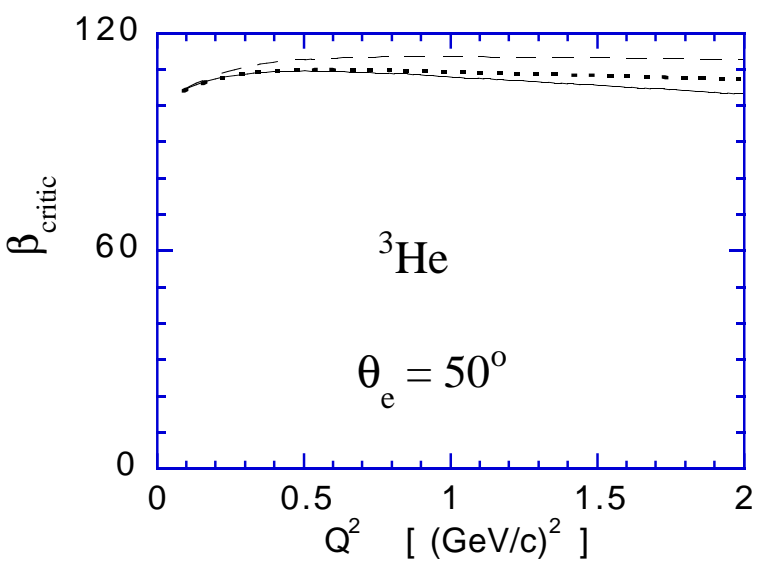

Figure 4. The polarization angle $\beta_{\text {critic }}$, where the proton contribution to the polarized cross section of ${ }^{3} \mathrm{He}$, at the qe peak, vanishes, vs $Q^{2}$. Solid line: Av14 + Coulomb interaction; dashed line: Av14 + Coulomb interaction + three-body forces; dotted line: RSCv8 + Coulomb interaction. (After [2]).

[1] C. E. Jones-Woodward et al., Phys. Rev. C 47 (1993) 110; C. E. Jones-Woodward et al., Phys. Rev. C 52 (1995) 1520; A. K. Thompson et al., Phys. Rev. Lett. 68 (1992) 2901; H. Gao et al., Phys. Rev. C 50 (1994) R546; J. O. Hansen et al., Phys. Rev. Lett. 74 (1995) 654.

[2] A. Kievsky, E. Pace, G. Salmè and M.Viviani: Phys. Rev. C 56 (1997) 64.

[3] J. Golak, S. Ishikawa, W. Glöckle, H. Witala, H. Kamada, D. Hüber, Conference Handbook of the XVth International Conference on Few-Body Problems in Physics, Groningen, July 22-26, 1997, p. 296.

[4] H. Gao et al., E-95-001 CEBAF approved experiment.

[5] R. B. Wiringa, R. A. Smith and T. A. Ainsworth, Phys. Rev. C 29 (1984) 1207.

[6] R. V. Reid, Ann. Phys. (N.Y.) 50 (1968) 411.

[7] H. T. Coelho, T. K. Das and M. R. Robilotta, Phys. Rev. C 28 (1983) 1812.

[8] S. A. Coon, M.D. Scadron, P.C. McNamee, B.R. Barret, D.W.E. Blatt and B.H.J. McKellar, Nucl. Phys. A317 (1979) 242; S. A. Coon and W. Glökle, Phys. Rev. C 23 (1981) 1790.

[9] C. Ciofi degli Atti, E. Pace and G. Salmè, Phys. Rev. C 46 (1992) R1591; Phys. Rev. C 51 (1995) 1108.

[10] R. W. Schültze and P.U. Sauer, Phys. Rev. C 48 (1993) 38.

[11] S. Galster et al., Nucl. Phys. B32 (1971) 221. 